\title{
Análisis descriptivo de adultos mayores mexicanos con enfermedad COVID-19
}

\section{COVID-19 in older adults Mexican, descriptive analysis}

\author{
Carla Paola Sánchez-Ríos, ${ }^{*}$ José Omar Barreto-Rodríguez, ${ }^{*}$ Gustavo Iván Centeno-Sáenz, ${ }^{*}$ Hazel Vázquez-Rojas*
}

*Instituto Nacional de Enfermedades Respiratorias Ismael Cosío Villegas, Ciudad de México, México.

RESUMEN. Introducción: Los adultos mayores pueden representar un grupo específico de pacientes de alto riesgo de desarrollar COVID-19 con deterioro clínico rápidamente progresivo. De hecho, en individuos mayores la inmunosenescencia y los trastornos comórbidos son más propensos a promover una tormenta de citocinas inducida por virus que resulte en insuficiencia respiratoria potencialmente mortal y compromiso multisistémico. Material y métodos: Se realizó un estudio descriptivo de la población geriátrica con enfermedad COVID-19 atendida en el Instituto Nacional de Enfermedades Respiratorias Ismael Cosío Villegas, Ciudad de México en el período de marzo a mayo de 2020. Se registraron 35 pacientes adultos mayores hospitalizados confirmados por RT-PCR para enfermedad COVID-19 en exudado nasofaríngeo. Se evaluó la distribución de los datos con la prueba de normalidad Shapiro-Wilks. Las variables nominales y ordinales se presentaron como porcentajes. Resultados: Se analizó una muestra total de 35 pacientes. $62.85 \%$ fueron masculinos $(n=22)$. Se documentó hipertensión arterial sistémica en $51.42 \%(n=18)$, obesidad en $48.57 \%(n=17)$ y diabetes mellitus en $34.28 \%(n=12)$. La gravedad de la enfermedad COVID-19 al momento del ingreso hospitalario fue: leve en $22.85 \%(n=8)$, moderada en $42.85 \%(n=15)$ y grave o crítica en $34.28 \%(n=12)$. De estos pacientes, $80 \%(\mathrm{n}=28)$ consultó después de presentar síntomas por más de cinco días. Los hallazgos tomográficos fueron patrón de vidrio deslustrado en 82.85\% ( $n=29)$, consolidación en $14.28 \%(n=5)$ y crazy paving en sólo un paciente $(2.8 \%)$. La mortalidad registrada en pacientes geriátricos enfermos de COVID-19 fue de $68.57 \%$. Conclusiones: Los adultos mayores ingresados en el Instituto Nacional de Enfermedades Respiratorias Ismael Cosío Villegas para manejo de COVID-19 fueron comórbidos en la mayoría de casos. El espectro leve de la enfermedad fue el menos frecuente. Estos pacientes buscan tardíamente atención médica posterior al inicio de síntomas, lo que es probable que impacte en la mortalidad; sin embargo, se requieren más estudios para confirmar estas aseveraciones.

Palabras clave: Adulto mayor, COVID-19, pronóstico.

Correspondencia:

Dra. Carla Paola Sánchez-Ríos

Instituto Nacional de Enfermedades Respiratorias Ismael Cosío

Villegas, Ciudad de México.

Correo electrónico: pao1144tost@gmail.com

Trabajo recibido: 05-VII-2020; aceptado: 15-IX-2020.

Citar como: Sánchez-Ríos CP, Barreto-Rodríguez JO, Centeno-Sáenz Gl, Vázquez-Rojas $\mathrm{H}$. Análisis descriptivo de adultos mayores mexicanos con enfermedad COVID-19. Neumol Cir Torax. 2020; 79 (4): 224-229. https:// dx.doi.org/10.35366/97964
ABSTRACT. Introduction: The elderly may represent a specific group of patients at high risk for developing COVID-19 with rapidly progressive clinical deterioration. In fact, in older individuals, immunosenescence and comorbid disorders are more likely to promote a virus-induced cytokine storm resulting in life-threatening respiratory failure and multisystem compromise. Material and methods: A descriptive study of the geriatric population with COVID-19 disease attended at the National Institute of Respiratory Diseases, Mexico City, Mexico in the period March-May 2020 was carried out. 35 hospitalized adult patients over 60 years were confirmed by RT PCR. for COVID-19 disease in pharyngeal exudate. Descriptive statistics were used and results were expressed using measures of central tendency. Results: A total sample of 35 patients was analyzed. $62.85 \%$ were male $(n=22)$. Systemic arterial hypertension was documented in $51.42 \%(n=18)$, obesity in $48.57 \%$ $(n=17)$ and diabetes mellitus in $34.28 \%(n=12)$. The severity of the COVID-19 disease at the time of hospital admission was: mild in $22.85 \%$ $(n=8)$, moderate in $42.85 \%(n=15)$ and severe or critical in $34.28 \%$ $(n=12) .80 \%(n=28)$ consulted after presenting symptoms for more than five days. The tomographic findings were ground glass pattern in $82.85 \%(n=29)$, consolidation in $14.28 \%(n=5)$ and «crazy paving» in only one patient $(2.8 \%)$. The mortality registered in geriatric patients with COVID-19 was $68.57 \%$. Conclusions: The geriatric population is considered vulnerable for the development of COVID-19 disease and for fatal outcomes. Patients with moderate disease should be closely monitored as they tend to eventually develop critical forms. The fatality of COVID-19 disease in older adult patients is high.

Keywords: Older adult, COVID-19, prognosis.
La nueva enfermedad por coronavirus (COVID-19) causada por el SARS-CoV-2 desde su inicio se ha propagado rápidamente. ${ }^{1,2}$ Los coronavirus representan un grupo heterogéneo de grandes virus de ARN de cadena sencilla, ampliamente distribuidos entre mamíferos y aves, agrupados en la familia de Coronaviridae. ${ }^{3}$ Los géneros de interés para los humanos son los coronavirus alfa y beta que componen la subfamilia de Coronavirinae, junto con los coronavirus gamma y delta, 
no reconocidos como patógenos humanos. ${ }^{4} \mathrm{~A}$ finales de la segunda década del siglo XXI, el mundo fue testigo de un brote de un nuevo coronavirus, designado SARS-CoV-2, y asignado taxonómicamente a las especies de coronavirus agudo severo relacionado con el síndrome respiratorio agudo (SARS-CoV), subgénero Sarbecovirus, género betacoronavirus. ${ }^{5,6}$ El término acuñado para referirse a la enfermedad relacionada con el SARS-CoV-2 es COVID-19. ${ }^{7}$ Las gotas y el contacto cercano se han reconocido como las principales vías de transmisión del virus SARS-CoV-2 en COVID- $19,{ }^{8}$ aunque la ruta fecal-oral no puede excluirse. ${ }^{9,10}$

El escenario clínico de COVID-19 es heterogéneo. De hecho, el espectro de la enfermedad varía desde síntomas leves como fiebre, tos seca y disnea hasta formas moderadas con síndrome de dificultad respiratoria aguda (SDRA) o bien formas, graves y críticas donde se presenta SDRA con necesidad de ventilación mecánica, o bien, sepsis y falla orgánica múltiple secundaria. ${ }^{11-13}$ Además, también se ha informado un curso asintomático, lo que hace que la contención de la infección sea más difícil.

Entre los pacientes infectados con SARS-CoV-2, varias afecciones están relacionadas con una mayor susceptibilidad al virus y una carga elevada de COVID-19. Al día de hoy la evidencia sugiere que la edad avanzada es el predictor más importante para el desenlace fatal. ${ }^{14}$

El envejecimiento en sí se ha asociado fuertemente con peores resultados, debido a los cambios fisiopatológicos que caracterizan el sistema respiratorio..$^{15}$ Según los datos epidemiológicos actuales, los pacientes adultos mayores infectados con SARS-CoV-2 muestran mayor riesgo de muerte en comparación con los pacientes más jóvenes. ${ }^{16-18}$

Los estudios que describen desenlace de infección por SARS-CoV-2 en adultos mayores cada vez es mayor. Wang et al. 2020 mencionan haber encontrado que el SARSCoV-2 causó una neumonía mucho más grave en los adultos mayores que en los pacientes más jóvenes, pues de los 339 pacientes adultos mayores con COVID-19 incluidos en su cohorte, más de $70 \%$ fueron graves o críticos con una tasa de letalidad de $19 \%{ }^{19}$

El objetivo de este estudio es describir la presentación de enfermedad COVID-19 de adultos mayores mexicanos internados en el Instituto Nacional de Enfermedades Respiratorias (INER) Ismael Cosío Villegas de marzo a mayo de 2020.

\section{MATERIAL Y MÉTODOS}

Este estudio se realizó en el INER Ismael Cosío Villegas. Fueron analizados de manera retrospectiva expedientes clínicos, imagenológicos y de microbiología de pacientes hospitalizados por enfermedad COVID-19. Se recabó la información necesaria para documentar variables sociodemográficas (edad, género, lugar de residencia, comorbilidades y tabaquismo) forma de enfermedad COVID-19, análisis paraclínicos y hallazgos tomográficos. Se trabajó con todos los expedientes de pacientes reclutados a conveniencia. Se eliminaron expedientes incompletos. La información se almacenó en una base de datos de Excel estratificando en función de la definición de las variables. Se evaluó la distribución de los datos con la prueba de normalidad Shapiro-Wilks. Los datos no paramétricos se reportaron con mediana. Las variables nominales y ordinales se presentaron como porcentajes.

\section{Definición de adulto mayor}

Según las Naciones Unidas se considera adulto mayor a toda persona mayor de 60 años en países en vías de desarrollo como México.

\section{Definición de enfermo COVID-19}

Paciente de cualquier edad que en los últimos siete días presente dos de los siguientes síntomas: tos seca, dolor de cabeza, fiebre y uno de los siguientes: escurrimiento nasal, disfagia, odinofagia, mialgias, conjuntivitis, rinorrea o dolor torácico más prueba de la reacción en cadena de la polimerasa de transcripción inversa inicial (RT-PCR-) SARS-CoV-2 positiva en laboratorio aprobado por el InDRE.

Definición de gravedad de enfermedad COVID-19 según la OMS:

- Enfermedad leve: paciente sintomático que se ajusta a la definición de caso de COVID-19, pero no presenta neumonía vírica ni hipoxia.

- Enfermedad moderada: neumonía con signos clínicos de neumonía (fiebre, tos, disnea, taquipnea), pero sin signos de neumonía grave, en particular $\mathrm{SpO}_{2} \geq 90 \%$ con aire ambiente.

- Enfermedad grave: neumonía grave, adolescente o adulto con signos clínicos de neumonía (fiebre, tos, disnea, taquipnea) más alguno de los siguientes: frecuencia respiratoria $>30$ inspiraciones/min, dificultad respiratoria grave o $\mathrm{SpO}_{2}<90 \%$ con aire ambiente.

\section{- Enfermedad crítica: SDRA.}

- SDRA leve: $200 \mathrm{mmHg}<\mathrm{PaO}_{2} / \mathrm{FiO}_{2} \mathrm{a} \leq 300 \mathrm{mmHg}$ (CON PEEP o CPAP $\geq 5 \mathrm{cmH}_{2} \mathrm{O}$ ).

- SDRA moderado: $100 \mathrm{mmHg}<\mathrm{PaO}_{2} / \mathrm{FiO}_{2} \leq 200$ $\mathrm{mmHg}$ (con PEEP $\geq 5 \mathrm{cmH}_{2} \mathrm{O}$ ).

- SDRA grave: $\mathrm{PaO}_{2} / \mathrm{FiO}_{2} \leq 100 \mathrm{mmHg}$ (con PEEP $\geq 5$ $\mathrm{CmH}_{2} \mathrm{O}$ ).

Septicemia: disfunción orgánica aguda y potencialmente mortal causada por una desregulación de la respuesta del 
huésped a una infección presunta o demostrada. Signos de disfunción orgánica: alteración del estado mental, disnea o taquipnea, $\mathrm{SpO}_{2}$ baja, oliguria, taquicardia, pulso débil, extremidades frías o hipotensión arterial, piel jaspeada, datos de coagulopatía en las pruebas de laboratorio, trombocitopenia, acidosis, hiperlactatemia o hiperbilirrubinemia.

Choque séptico: lactato sérico $>2 \mathrm{mmol} / \mathrm{L}$ e hipotensión persistente que pese a la reposición de la volemia necesita vasopresores para mantener una TA media $\geq 65 \mathrm{mmHg}$.

\section{Metodología de RT-PCR SARS-CoV-2 INER}

Se utiliza la prueba Xpert SARS-CoV-2, que es una prueba de RT-PCR en tiempo real para la detección cualitativa de ácidos nucleicos del virus SARS-CoV-2 en muestras de hisopos nasofaríngeos, hisopos nasales o lavado/aspirado nasal de personas con sospecha de infección COVID-19. Los resultados permiten identificar el ARN de SARS-CoV-2. Los resultados positivos indican la presencia de ARN de SARS-CoV-2.

\section{RESULTADOS}

Se analizó una muestra total de 35 pacientes adultos mayores hospitalizados con enfermedad COVID-19 confirmada por RT-PCR de exudado nasofaríngeo en el período de estudio. El promedio de edad fue de 69 años (+ DE 6.7). De éstos, $62.85 \%$ fueron masculinos $(n=22)$. Las alcaldías de donde provenían la mayor cantidad de pacientes se registraron de la siguiente manera: Iztapalapa, Xochimilco, Coyoacán y Cuauhtémoc. Respecto a las comorbilidades presentadas por los pacientes se documentó hipertensión arterial sistémica en $51.42 \%(n=18)$, obesidad en $48.57 \%$ $(n=17)$ y diabetes mellitus en $34.28 \%(n=12)$. No se reportó cáncer, enfermedad renal crónica o neumopatías. La exposición a tabaco y humo de leña fue documentada en rango de riesgo de enfermedad pulmonar obstructiva crónica (EPOC) por índice tabáquico e índice de exposición a humo de leña en $25 \%(n=9)$ y $20 \%$ (7), respectivamente; sin embargo, ningún paciente tenía el diagnóstico espirométrico de EPOC al ingreso (Figura 1).

La prueba de RT-PCR para SARS-CoV-2 de exudado nasofaríngeo inicial se reportó positiva en 100\% (35) de pacientes. El promedio de saturación por oximetría de pulso inicial fue de $73 \%$ (+ DE 17.2) al aire ambiente y el índice de masa corporal (IMC) promedio al ingreso fue de 30 (+ DE 4.18). La graduación de la enfermedad COVID-19 al momento del ingreso hospitalario fue leve en $22.85 \%$ $(n=8)$; moderada en $42.85 \%(n=15)$, y grave o crítica en $34.28 \%(n=12)$ (Figura 2). $80 \%(n=28)$ consultó después de presentar síntomas por más de cinco días.

En el análisis paraclínico se documentó leucocitosis en $40 \%(n=14)$ con relación neutrófilo/linfocito promedio de
13.3 ( \pm DE 12). En 68.57\% ( $n=24)$ se documentó linfopenia. En el registro tomográfico inicial se evidenció patrón predominantemente de vidrio deslustrado en $82.85 \%$ $(n=29)$, con predominio de consolidación en $14.28 \%$ $(n=5)$ y patrón con predominio de crazy paving en sólo un paciente $(2.8 \%)$.

La mortalidad registrada en pacientes adultos mayores enfermos de COVID-19 fue de 68.57\% $(n=24)$ (Figura 3).

\section{DISCUSIÓN}

Sabemos que la inmunosenescencia representa una característica reconocida del envejecimiento. A medida que avanza la edad, se ha informado alteración de las vías innatas y adaptativas del sistema inmunológico. Además, los adultos mayores exhiben una producción continua de mediadores inflamatorios y citocinas. ${ }^{20-22}$ La función ciliar aberrante y las anomalías ultraestructurales ciliares podrían poner en peligro la eliminación exitosa de las partículas del virus SARS-CoV-2 en adultos mayores contribuyendo a la vulnerabilidad a la enfermedad COVID-19 en este grupo.

Además de una mayor susceptibilidad debido al envejecimiento, los estudios epidemiológicos han revelado diferencias específicas por sexo en la incidencia y mortalidad en humanos después de la infección por SARS-CoV y SARS-CoV-2. El género masculino ha experimentado mayor mortalidad en comparación con el femenino. ${ }^{23-26}$ En nuestro estudio, el género masculino fue el más afectado por la infección y también representó mayor porcentaje en el grupo de desenlaces fatales (66.66\%).

Curiosamente, este aumento dependiente del género en la gravedad de la enfermedad después de la infección por coronavirus es más pronunciado con la edad avanzada. De hecho, Jin et al. demostraron recientemente que la edad

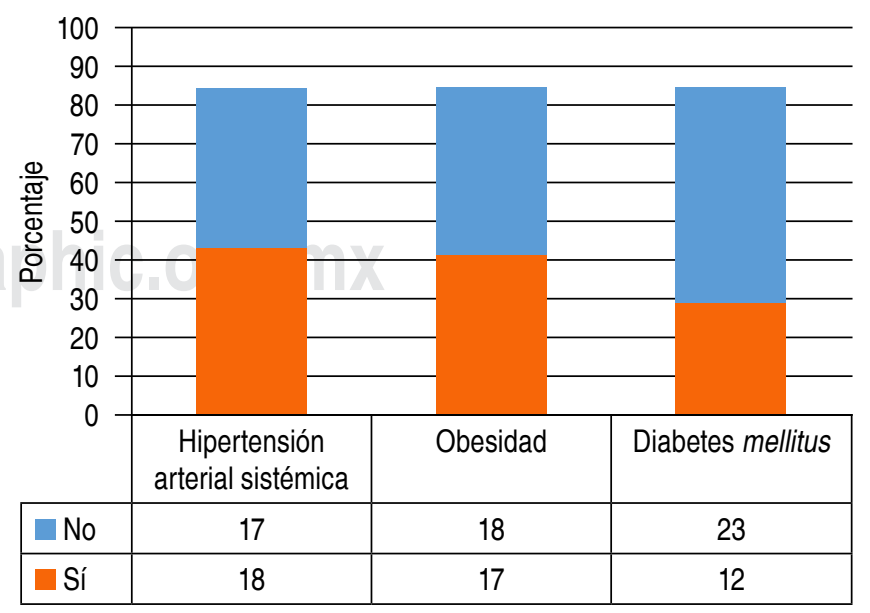

Figura 1: Principales comorbilidades en adultos mayores enfermos de COVID-19. 


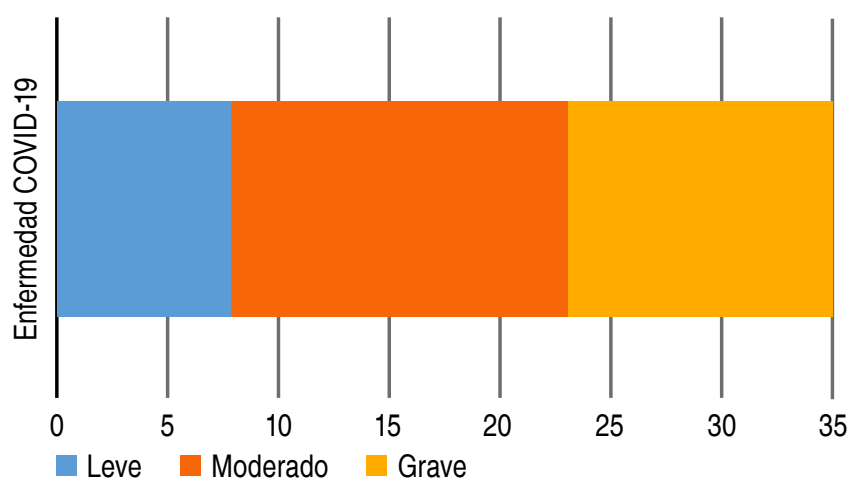

Figura 2: Espectro de gravedad de la enfermedad COVID-19 de adultos mayores hospitalizados en el INER.

avanzada es un factor de riesgo predominante de gravedad y morbilidad en pacientes con COVID-19. Mientras que los hombres y las mujeres tienen la misma susceptibilidad a COVID-19, los pacientes varones pueden ser más propensos a morir. En nuestro estudio, si bien la metodología no puede demostrar esta aseveración, descriptivamente por frecuencia sí se evidenció del total de desenlaces fatales mayor cantidad en enfermos de género masculino.

Liu et al. ${ }^{27}$ analizaron las características clínicas de pacientes de edad avanzada con COVID-19 para identificar las diferencias entre pacientes de edad avanzada y pacientes más jóvenes. Los síntomas más comunes en ambos grupos fueron fiebre, tos y esputo; los síntomas concomitantes menos comunes fueron secreción nasal, dolor de cabeza y diarrea. La puntuación del índice de gravedad de la neumonía (PSI) del grupo de adultos mayores fue mayor en comparación con el grupo de jóvenes. En nuestro estudio, el porcentaje de pacientes que documentó mayor temporalidad de síntomas mostró también mayor afección tomográfica y desenlaces fatales.

Los estudios en población geriátrica han documentado que los análisis de sangre de rutina se usan ampliamente para diagnosticar y controlar el curso de COVID-19 en adultos mayores debido a que pueden manifestar síntomas inusuales o no presentar la sintomatología típica con elevación de temperatura franca. En un estudio retrospectivo no se observaron diferencias significativas en el recuento de glóbulos blancos, la relación de neutrófilos, la procalcitonina, el nivel de hemoglobina, las plaquetas y la creatinina sérica en el grupo de pacientes de edad avanzada. ${ }^{26}$ En nuestro estudio se evidenció que la población geriátrica tiende a presentar linfopenia y aumento de dímero, tal como lo menciona Huang (2020) en su estudio. ${ }^{2}$ Nosotros documentamos una mediana de relación neutrófilo/linfocito de 11 , procalcitoninas positivas al ingreso en $28.5 \%$ sin documentar coinfección bacteriana. Covino et al. reportaron factores de riesgo independientes asociados con muerte en población geriátrica de COVID-19, donde LDH mayor de $464 \mathrm{U} / \mathrm{L}$ y la hipoxemia $\left(\mathrm{PO}_{2}\right.$ menor de $90 \mathrm{mmHg}$ ) fueron factores descritos. En nuestro estudio 100\% de los pacientes que fallecieron, documentaron al ingreso hipoxemia y saturación inicial menor de $90 \%$ por oximetría de pulso y $62.5 \%$ presentaron $\mathrm{LDH}$ inicial mayor de $464 \mathrm{U} / \mathrm{L}^{28}$

Entre las herramientas de diagnóstico mencionadas anteriormente, las imágenes de tórax representan la piedra angular para detectar anomalías pulmonares durante la infección viral. ${ }^{29,30}$ Se ha documentado que la tomografía computarizada del tórax tiene mayor sensibilidad para el diagnóstico de COVID-19 que la RT-PCR. Las características típicas de la presentación de tomografía torácica por COVID-19 incluyen la opacificación bilateral de vidrio esmerilado multilobar, con una distribución periférica o posterior, principalmente en los lóbulos inferiores. ${ }^{31}$ Tal como lo reportamos en nuestro estudio, el hallazgo tomográfico típico predominante fue el vidrio deslustrado multilobar. La presentación de imagen inicial atípica de opacidades consolidadas superpuestas en vidrio esmerilado se puede encontrar en un número menor de casos, principalmente en la población de edad avanzada. Consideramos que dentro de la evolución tomográfica de la enfermedad COVID-19, el hecho de que la mayoría de pacientes de nuestra población consultara con más de cinco días de evolución, contribuyó a que el patrón de consolidación se presentara con más frecuencia que en otras cohortes.

Del porcentaje de infectados por SARS-CoV-2 con enfermedad COVID-19 moderada, 73.3\% mostró deterioro clínico durante el internamiento ameritando manejo avanzado de la vía aérea. En total, sumado al porcentaje de enfermos COVID-19 grave inicial, $65.71 \%$ se encontraba en manejo de ventilación mecánica bajo cuidados de enfermo crítico en unidad de cuidados intensivos. Este deterioro clínico presentado por adultos mayores infectados por coronavirus contribuye al rápido agotamiento de camas para manejo

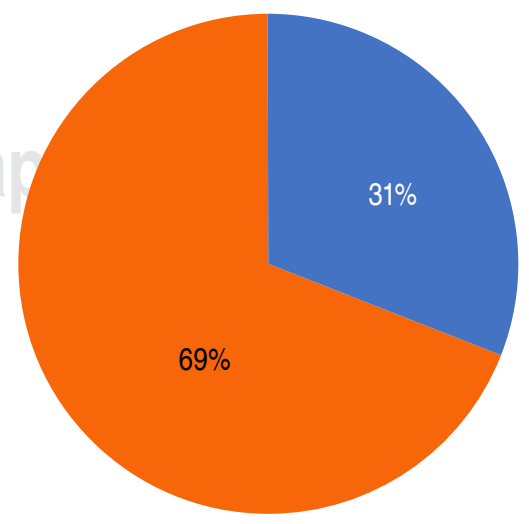

Egreso por mejoría

Egreso por defunción

Figura 3: Desenlace de adultos mayores con enfermedad COVID-19 hospitalizados en el INER. 
de cuidados intensivos, además de la escasa probabilidad de supervivencia. Por todo lo comentado con anterioridad, se debe asegurar que los cuidados paliativos más efectivos soporten, tanto física como psicológicamente, el final de la vida de estos enfermos. ${ }^{31} \mathrm{Al}$ día de hoy se recomienda que las instituciones de atención médica incluyan protocolos de cuidados paliativos en las vías de atención de COVID-19 para enfermos no recuperables. ${ }^{32}$

\section{CONCLUSIONES}

Los adultos mayores ingresados en el INER para manejo de COVID-19 fueron comórbidos en la mayoría de los casos. El espectro leve de la enfermedad fue el menos frecuente. Estos pacientes buscan tardíamente atención médica posterior al inicio de síntomas, lo que es probable que impacte en la mortalidad; sin embargo, se requieren más estudios para confirmar estas aseveraciones.

\section{REFERENCIAS}

1. World Health Organization. Coronavirus disease 2019 (COVID-19) situation report-97. Geneva: World Health Organization; 2020.

2. Huang $C$, Wang Y, Li X, Ren L, Zhao J, Hu Y, et al. Clinical features of patients infected with 2019 novel coronavirus in Wuhan, China. Lancet. 2020; 395(10223): 497-506. https://doi.org/10.1016/s01406736(20)30183-5

3. Masters PS. The molecular biology of coronaviruses. Adv Virus Res. 2006;66:193-292. https://doi.org/10.1016/s0065-3527(06)66005-3

4. Kang S, Peng W, Zhu Y, Lu S, Zhou M, Lin W, et al. Recent progress in understanding 2019 novel coronavirus (SARS-CoV-2) associated with human respiratory disease: detection, mechanisms and treatment. Int J Antimicrob Agents. 2020;55(5):105950. https://doi.org/10.1016/j. ijantimicag.2020.105950

5. Gorbalenya AE, Baker SC, Baric RS, et al; Coronaviridae Study Group of the International Committee on Taxonomy of Viruses. The species severe acute respiratory syndrome-related coronavirus: classifying 2019-nCoV and naming it SARS-CoV-2. Nat Microbiol. 2020;5(4):536544. https://doi.org/10.1038/s41564-020-0695-z

6. Zhou P, Yang XL, Wang XG, Hu B, Zhang W, Si HR, et al. A pneumonia outbreak associated with a new coronavirus of probable bat origin. Nature. 2020;579(7798):270-273. https://doi.org/10.1038/s41586-0202012-7

7. World Health Organization. Novel coronavirus (2019-nCoV) situation report-22. Geneve: World Health Organization; 2020. https://apps. who.int/iris/handle/10665/330991

8. World Health Organization. Modes of transmission of virus causing COVID-19: implications for IPC precaution recommendations. Geneva: World Health Organization; 2020. p. 10-12.

9. Xu Y, Li X, Zhu B, Liang H, Fang Ch, Gong Y, et al. Characteristics of pediatric SARS-CoV-2 infection and potential evidence for persistent fecal viral shedding. Nat Med. 2020;26(4):502-505. https://doi. org/10.1038/s41591-020-0817-4

10. Hindson J. COVID-19: faecal-oral transmission? Nat Rev Gastroenterol Hepatol. 2020;17(5):259. https://doi.org/10.1038/s41575-0200295-7
11. Chen N, Zhou M, Dong X, QuJ, Gong F, Han Y, etal. Epidemiological and clinical characteristics of 99 cases of 2019 novel coronavirus pneumonia in Wuhan, China: a descriptive study. Lancet. 2020;395(10223):507513. https://doi.org/10.1016/s0140-6736(20)30211-7

12. Zhu N, Zhang D, Wang W, Li X, Yang B, Song J, et al. A novel coronavirus from patients with pneumonia in China, 2019. N Engl J Med. 2020;382(8):727-733. https://doi.org/10.1056/nejmoa2001017

13. Zhou F, Yu T, Du R, Fan G, Liu Y, Liu Z, et al. Clinical course and risk factors for mortality of adult inpatients with COVID-19 in Wuhan, China: a retrospective cohort study. Lancet. 2020;395(10229):10541062. https://doi.org/10.1016/s0140-6736(20)30566-3

14. Nishiura H, Kobayashi T, Miyama T, Suzuki A, Jung SM, Hayashi $\mathrm{K}$, et al. Estimation of the asymptomatic ratio of novel coronavirus infections (COVID-19). Int J Infect Dis. 2020;94:154-155. https://doi. org/10.1016/ji.jid.2020.03.020

15. Libertini G, Corbi G, Cellurale M, Ferrara N. Age-related dysfunctions: evidence and relationship with some risk factors and protective drugs. Biochemistry (Mosc). 2019;84(12):1442-1450. https://doi.org/10.1134/ s0006297919120034

16. Lionakis N, Mendrinos D, Sanidas E, Favatas G, Georgopoulou M. Hypertension in the elderly. World J Cardiol. 2012;4(5):135-147. https:// doi.org/10.4330/wjc.v4.15.135

17. Suastika K, Dwipayana P, Saraswati IMR, Kuswardhani T, Astika N, Putrawan IB, et al. Relationship between age and metabolic disorders in the population of Bali. J Clin Gerontol Geriatr. 2011;2:47-52. https:// doi.org/10.1016/j.jcgg.2011.03.001

18. Palmieri L, Andrianou X, Barbariol P. Characteristics of SARS-CoV-2 patients dying in Italy report based on available data on April 16th, 2020. Access date: 2020 November 19. Available in: https://www. epicentro.iss.it/en/coronavirus/bollettino/Report-COVID-2019_29_ april_2020.pdf

19. Wang L, He W, Yu X, Hu D, Bao M, Liu H, et al. Coronavirus disease 2019 in elderly patients: characteristics and prognostic factors based on 4-week follow-up. J Infect. 2020;80(6):639-645. https://doi. org/10.1016/j.jinf.2020.03.019

20. Aw D, Silva AB, Palmer DB. Immunosenescence: emerging challenges for an ageing population. Immunology. 2007;120(4):435-446. https://doi.org/10.1111/j.1365-2567.2007.02555.x

21. Longobardi L, Giorgio AD, Perrotta F, Costigliola A, Cerqua FS, Cioffi $G$, et al. Bronchial asthma in the elderly patient. JGG. 2016;64:55-65.

22. Mollica M, Nicolai A, Maffucci R, Gioia MR, Paoli G, Grella E, et al. Obstructive sleep apnea and cardiovascular risks in the elderly population. JGG. 2018;66(3):149-155.

23. Karlberg J, Chong DS, Lai WY. Do men have a higher case fatality rate of severe acute respiratory syndrome than women do? Am J Epidemiol. 2004;159(3):229-231. https://doi.org/10.1093/aje/kwh056

24. Leong HN, Earnest A, Lim HH, Chin CF, Tan C, Puhaindran ME, et al. SARS in Singapore--predictors of disease severity. Ann Acad Med Singap. 2006;35(5):326-331.

25. Alghamdi IG, Hussain II, Almalki SS, Alghamdi MS, Alghamdi MM, El-Sheemy MA. The pattern of Middle East respiratory syndrome coronavirus in Saudi Arabia: a descriptive epidemiological analysis of data from the Saudi Ministry of Health. Int J Gen Med. 2014;7:417423. https://doi.org/10.2147/ijgm.s67061

26. Conti P, Younes A. Coronavirus COV-19/SARS-CoV-2 affects women less than men: clinical response to viral infection. J Biol Regul Homeost Agents. 2020;34(2):339-343. https://doi.org/10.23812/ editorial-conti-3 
27. Liu K, Chen Y, Lin R, Han K. Clinical features of COVID-19 in elderly patients: a comparison with young and middle-aged patients. J Infect. 2020;80(6):e14-e18. https://doi.org/10.1016/j.jinf.2020.03.005

28. Covino M, De Matteis G, Santoro M, Sabia L, Simeoni B, Candelli $M$, et al. Clinical characteristics and prognostic factors in COVID-19 patients aged $\geq 80$ years. Geriatr Gerontol Int. 2020;20(7):704-708. https://doi.org/10.1111/ggi.13960

29. Lee EYP, Ng MY, Khong PL. COVID-19 pneumonia: what has CT taught us? Lancet Infect Dis. 2020;20(4):384-385. https://doi. org/10.1016/s1473-3099(20)30134-1

30. Rinaldi L, Milione S, Fascione MC, Pafundi PC, Altruda C, Di Caterino $\mathrm{M}$, et al. Relevance of lung ultrasound in the diagnostic algorithm of respiratory diseases in a real-life setting: a multicentre prospective study. Respirology. 2010;25(5):535-542. https://doi.org/10.1111/ resp.13659

31. World Health Organization. Integrating palliative care and symptom relief into responses to humanitarian emergencies and crises: a WHO guide. Geneva: World Health Organization; 2018.

32. Nacoti M, Ciocca A, Giupponi A, Brambillasca P, Lussana F, Pisano $M$, et al. At the epicenter of the Covid-19 pandemic and humanitarian crises in Italy: changing perspectives on preparation and mitigation. NEJM Catal Innov Care Deliv. 2020;1:1-5.

Conflicto de intereses: Los autores declaran no tener conflicto de intereses. 